que les projections sur papier mince sont appelées à rendre de réels services, en raison de leur bas prix, aux instituteurs et conférenciers qui ont pour but de vulgariser la botanique élémentaire.

Il est donné lecture de la communication suivante :

\title{
Recherches physiologiques sur le mouvement des étamines des Berbéridées,
}

\author{
PAR M. PAUL DOP.
}

Peu de questions ont été l'objet d'un aussi grand nombre de publications que l'étude des mouvements dits provoqués chez les végétaux. Et pourtant la question est restée des plus obscures. La preuve en est dans les faits suivants : pour Pfefrer $(39,42)$ le mouvement des étamines de Cynarées ou de Berbéridées s'accompagnerait de l'expulsion d'une gouttelette d'eau; or, il y a déjà trente ans, Heckec (22) démontrait l'inanité de cette assertion. Des observations plus récentes de Chauveaud (6) et de moi ont confirmé la manière de voir d'Heckel. Cependant la théorie de la goutte d'eau de Pfefrer est devenue classique et elle figure encore dans la dernière édition du traité de physiologie du savant allemand.

Une autre cause de la confusion qui règne dans nos connaissances sur les mouvements provoqués vient de la méthode mème, que certains auteurs ont employée dans leurs recherches. Ils ont cru que l'anatomie seule permettrait de résoudre les problèmes physiologiques; et ce faisant ils ont édifié des hypothèses ingénieuses et intéressantes, mais auxquelles manque le contrôle de l'expérience. Le lecteur qui désirerait avoir une idée de cette méthode et de ses résultats, n'aura qu'à parcourir le traité d'anatomie physiologique d'Habercandt (20).

De tout cela il résulte que c'est par l'expérience que les problèmes de physiologie doivent se traiter. Aussi c'est par l'expérience que j'ai abordé l'étude des mouvements des étamines des Berbéridées, et ce sont ces expériences faites dans les années 1905 et 1906 que je vais exposer. Ce travail débutera donc par une partie purement expérimentale; il se terminera par une 
étude critique, et par l'exposé de l'hypothèse qui, dans l'état actuel de la science, paraît la plus compatible avec les faits.

\section{$1^{\circ}$ Analyse graphique du mouvement.}

Quand on étudie un mouvement, la première chose à faire es de l'enregistrer. Il semble que cette idée pourtant bien simple ait échappé aux botanistes qui se sont occupés des Berbéridées. A ma connaissance, en effet, je n'ai rencontré dans la littérature botanique que deux applications de la méthode d'enregistrement. DEwèvre et Bordage (12) ont enregistré photographiquement les mouvements de circumnutation, et Risa Scotr (44) par des procédés cinématographiques, signalés d'ailleurs par Pfeffer, a étudié les mouvements des fleurs de Sparmannia africana.

Cee n'est pas la méthode cinématographique que j'ai employée, mais purement et simplement l'inscription directe du mouvement sur un cylindre enregistreur. Voici ma technique (fig. 1). Un fil de bambou st est collé avec de la cire blanche, le long du tiers supérieur de l'étamine et sur sa face externe. L'extrémité de ce fil vient s'appuyer sur un cylindre enregistreur. Quand l'étamine se meut, le fil est entraîné et trace sur le cylindre une courbe $c$ qui représente le mouvèment de l'extrémité supérieure de l'étamine en fonction du temps.

La difficulté réside dans les conditions suivantes à remplir : le stylet de bambou, à raison de son inertie, doit être aussi léger que possible et cependant assez long pour que la courbe que décrirait son extrémité libre sur un cylindre immobile se rapproche d'une ligne droite. Étant donné que le déplacement du sommet de l'étamine est de 20 à $30^{\circ}$, dans la plupart des Mahonia et des Berberis, une longueur de stylet de 4 centimètres remplit pratiquement les conditions nécessaires. Il faut ensuite disposer l'étamine et le stylet dans une orientation telle que, le cylindre enregistreur étant immobile, la ligne que décrit le sommet du stylet dans son mouvement soit une génératrice du cylindre. D'autre part pour se placer dans des conditions normales, il y a lieu d'enregistrer le mouvement d'une étamine n'ayant subi aucune mutilation, par conséquent encore fixée sur la fleur. Pour cela je prends une fleur dont j’échancre, d'un coup de ciseaux, les enveloppes. Cette fleur restant fixée par son 
pédoncule à un rameau plongeant dans de l'eau, est maintenue par une pince $p$, capable de s'orienter dans les trois plans de l'espace. Gràce à cette pince, et après tâtonnements et essais préliminaires, quelquefois délicats, il est possible de placer dans une position convenable par rapport au cylindre enregistreur, celle des six étamines à laquelle est collé le fil de bambou.

Il faut maintenant déterminer le mouvement. L'excitation électrique (voir page 559) le permet très facilement. La pince $p$ qui maintient la fleur est reliée à l'un des pôles d'une pile, et un fil de platine $f$ touchant l'anthère à l'autre pôle. Dans le circuit est intercalé un interrupteur et un signal électrique de Marey, permettant de repérer les temps. Il suffit d'établir le courant, la fermeture détermine le mouvement et le stylet de bambou inscrit le mouvement sur le cylindre.

Résultats. - Je décrirai le mouvement des étamines de Mahonia japonica, observé à la température de 18 degrés. Dans le mouvement je distinguerai deux périodes : la période d'aller correspondant au rabattement de l'étamine sur le pistil, et la période de retour dans laquelle l'étamine revient à sa position primitive.

a) Période d'aller ou d'énergie croissante. - Ainsi qu ill résulte de l'examen de la courbe ci-jointe (fig. 2), on voit que le mouvement se produit après une période d'excitation latente d'environ 1 dixième de seconde. La première phase du mouvement est brusque; en un dixième de seconde, le stylet subit en effet un déplacement de 5 millimètres, puis le mouvement se ralentit, $2 \mathrm{~mm}$. 5 sont parcourus en 4 dixièmes de seconde, et le dernier demi-millimètre nécessite encore un temps de 4 dixièmes de seconde. Ainsi donc le déplacement total étant do 8 millimètres, la durée du mouvement d'aller a été de 9 dixièmes de seconde, ou en tenant compte de la période d'excitation latente, d'une seconde environ. Ce mouvement est donc beaucoup plus lent que la contraction musculaire qui dure à peine un centième de seconde.

b) Période de retour ou d'énergie décroissante. - La période de retour a duré dix-sept minutes. Pendant les dix à douze premières secondes l'étamine reste immobile, dans la position de rabattement complet, puis elle revient en arrière très 
P. DOP. - MOUVEMENT DES ÉTAMINES DES BERbÉRIDÉES.

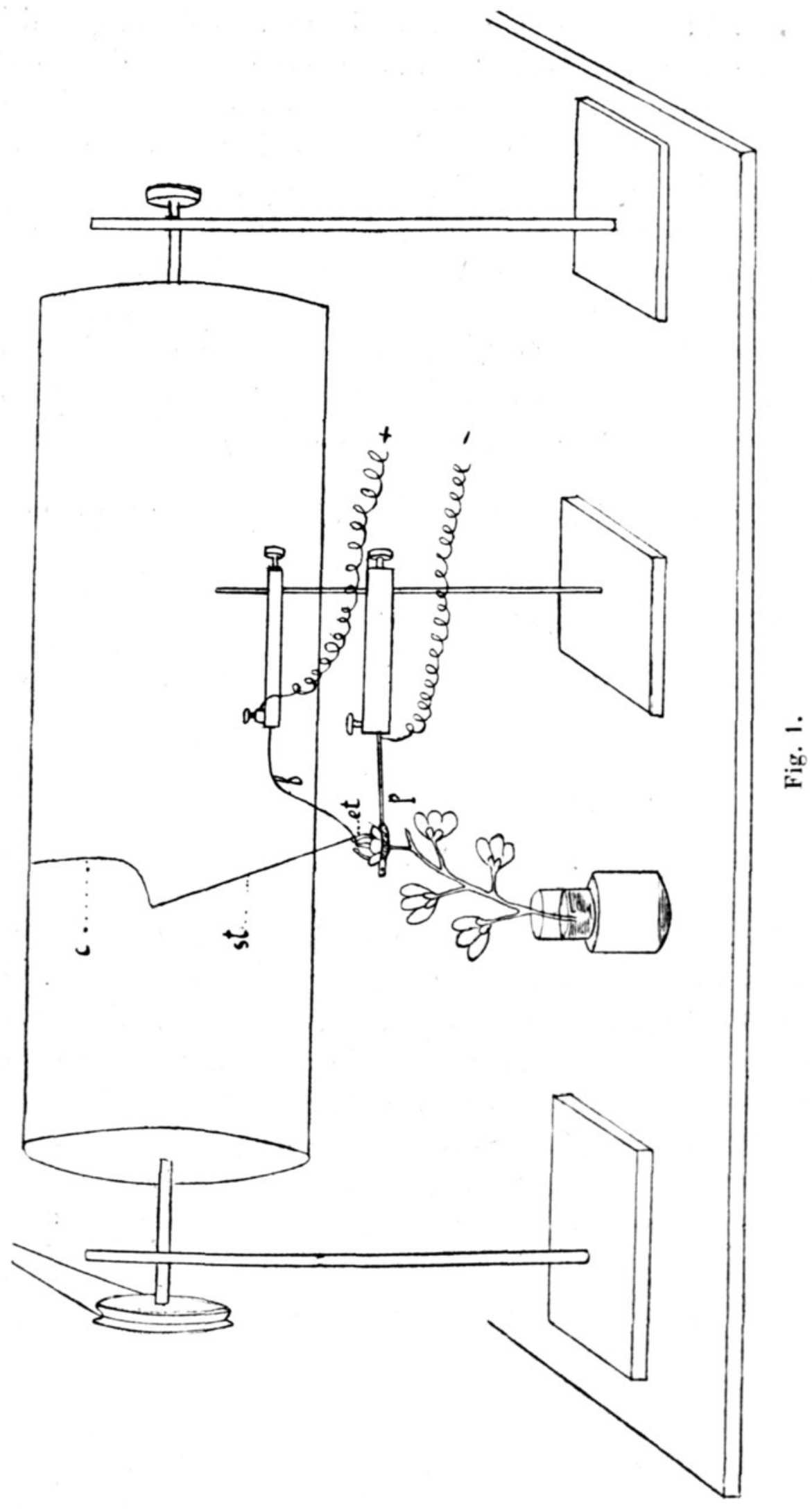

lentement. Pendant les sept premières minutes, le stylet recule 
de $\breve{5} \mathrm{~mm}$. 5 ; et enfin pour revenir à sa position primitive, c'est-à-dire pour parcourir $2 \mathrm{~mm}$. ̋̋, l'étamine met dix minutes. Ainsi donc la courbe représentative descendrait assez rapidement pendant les sept premières minutes, puis après elle devient pour ainsi dire asymptote à l'axe des temps.

En résumé les diverses phases du mouvement des étamines des Berbéridées se succèdent dans l'ordre suivant. Une période d'excitation latente qui dure un dixième de seconde, une période d'énergie croissante ou d'aller brusque et durant neuf dixièmes de seconde, une phase d'immobilité de dix à douze secondes, et enfin un retour lent $\dot{a}$ la position primitive, qui dure dix-sept minutes.

Cherchons maintenant à exciter l'étamine avant qu'elle soit

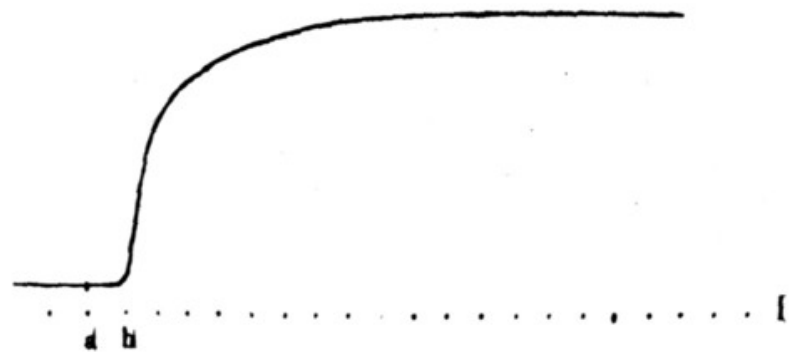

Fig. 2.

complètement revenue à sa position de repos. On constate que l'étamine reste insensible pendant les sept premières minutes de son mouvement de retour. Mais après ces sept minutes, alors que son sommet a encore un chemin de $2 \mathrm{~mm}$. 5 à accomplir, elle est de nouveau excitable et peut se rabattre sur le stigmate. Il résulte de cela qu'il est impossible de produire un tetanos proprement dit. Tout ce que l'on peut faire par des excitations répétées, c'est de supprimer la période de retour la plus lente, mais un intervalle minima de sept minutes est nécessaire pour que deux excitations successives soient possibles. D'ailleurs au bout de trois ou quatre excitations ainsi répétées, l'étamine se fatigue, les courbes d'aller s'affaissent et une longue période de repos devient nécessaire entre deux excitations successives.

L'étude analytique du mouvement des étamines des Berbéridées, établit d'une façon nette l'existence de trois périodes successives : une période d'excitation latente, une période d'énergie 
croissante et enfin une période d'énergie décroissante. Ce résultat est conforme aux recherches publiées par $\mathbf{M A C}_{\mathbf{A}} \mathrm{F}_{\mathbf{A R}-}$ Lane (31) sur des Oxalis, des Drosera, des Mimosa, en un mot sur les végétaux dont les feuilles réagissent par une courbure aux excitations. Essentiellement la succession des phénomènes est la même, et consiste en une période latente, une contraction et une expansion. $\mathrm{M}_{\mathrm{AC}} \mathrm{F}_{\mathrm{ARLANE}}$ conclut donc à l'analogie entre ces phénomènes et ceux qui se produisent dans la vie animale. Cependant il y a lieu, à mon sens, de noter qu'entre les phénomènes de contraction musculaire par exemple et les mouvements des étamines, il existe une différence importante, tenant à la lenteur de ces derniers, par rapport à la courte durée des premiers.

\section{$2{ }^{\circ}$ ÉLeGtro-Physiologie.}

L'excitation électrique des étamines des Berbéridées a été l'objet d'un certain nombre de travaux, dont les plus importants sont dus à Kabsch (25) et à HECKeL (22).

$\mathbf{K}_{\mathrm{ABSCH}}$ employa les courants induits fournis par une bobine de Rhumkorff; il constata la production du mouvement, mais il admit faussement que seul un courant capable d'anéantir la sensibilité peut produire le mouvement. НескеL reprit ces expériences en employant soit des courants continus, soit des courants induits. Il rectifia l'opinion précédente de $\mathrm{K}_{\mathrm{ABSCH}}$, mais il admit que dans le cas d'un courant continu, l'étamine reste contractée pendant tout le passage du courant. Depuis le travail d'HескеL, l'électro-physiologie des étamines de Berbéridées n'a fait aucun progrès, de telle sorte que la question méritait d'être reprise par des méthodes plus perfectionnées.

\section{Technique.}

On peut, pour étudier l'action des courants électriques sur les étamines mobiles, employer le dispositif de Iürgensen (24), qui consiste essentiellement en deux électrodes métalliques sur lesquelles reposent la base et le sommet d'une étamine sectionnée et maintenue dans une atmosphère humide. Pour ma part, j'estime qu'il vaut mieux opérer sur une étamine fixée à la 
fleur, en mettant, comme le faisait Heckes, le pédoncule floral en relation avec un pôle de la pile et l'anthère avec l'autre pôle. C'est le dispositif que j'ai employé pour enregistrer le mouvement des étamines mues électriquement.

Ces méthodes ont l'inconvénient d'ètre infidèles, car le contact est souvent mauvais entre l'électrode et l'étamine.

J'ai donc imaginé un autre procédé que j'ai exposé dans une Note antérieure (13) et qui consiste essentiellement à assurer le contact entre l'étamine et les électrodes, en imprégnant les membranes cellulaires de la solution d'un électrolyte. J'ai indiqué qu'il suffisait pour cela de placer dans la fleur une goutte de dissolution saline. En plaçant ensuite une électrode en platine au contact du stigmate et l'autre au contact de l'anthère, on peut très facilement produire et étudier les mouvements déterminés par l'excitation électrique.

\section{a). Courants continus.}

Résultats. - L'établissement d'un courant continu détermine le rabattement de l'étamine placée dans le circuit. Mais contrairement à l'opinion d'HЕскEL, l'étamine ne reste pas rabattue sur le stigmate pendant le passage du courant; elle revient à sa position de repos, en décrivant la courbe de retour que j'ai exposée dans le chapitre précédent. L'expérience ne laisse à ce sujet aucun doute, et il est possible d'énoncer la loi suivante :

La fermeture, c'est-à-dire l'établissement du courant, détermine un mouvement, dès que le courant est établi, létat de mouvement cesse.

L'ouverture, c'est-à-dire l'interruption du courant passant dans une étamine revenue à l'état de repos, ne m’a pas paru déterminer de mouvement; de telle sorte que contrairement à ce qui se passe dans les muscles, la secousse de fermeture est seule capable de déterminer le mouvement.

Le résultat est le même, que la fermeture ait lieu sur l'anode ou sur la cathode, et les courbes obtenues dans les deux cas sur le cylindre enregistreur sont comparables.

Quelle est l'intensité minima nécessaire pour produire le mouvement. J'ai trouvé que cette intensité variait entre trois 
et six millièmes d'ampère. C'est-à-dire qu'elle est du mème ordre de grandeur que celle qui est nécessaire à la contraction musculaire.

Enfin, j'ai pu m'assurer que KABsch était dans l'erreur quand il disait qu'une étamine excitée électriquement devenait ensuite insensible. En réalité, elle se comporte exactement comme une étamine excitée mécaniquement, c'est-à-dire qu'elle redevient mobile, sept minutes après la première excitation.

\section{b). Courants induits.}

Le chariot de Dubois Raymond permet facilement de réaliser de nombreuses expériences sur les fleurs de Mahonia ou de Berberis. Comme Kabsch et Heckel l'avaient constaté, les chocs d'induction provoquent le mouvement. Est-il possible de produire gràce à eux un état tétanique véritable? On peut à cette question répondre non, car l'intervalle de sept minutes est absolument nécessaire au retour de l'excitabilité.

\section{c). Champs électriques.}

Il est donc maintenant possible d'admettre que les courants continus ou induits déterminent le mouvement des étamines de Berbéridées en suivant des lois très simples. Je me suis posé la question de savoir si les mouvements des étamines pourraient être déterminés par des variations lentes ou brusques dans un champ électrique. Pour cela, j'ai placé des fleurs de Mahonia entre les deux boules d'une machine de Wimhurst. En faisant varier l'intensité du champ électrique, soit en déplaçant la fleur, soit en faisant varier la vitesse de rotation des plateaux, aucun mouvement ne s'est produit. Le résultat de mes expériences a donc toujours été négatif, et quoiqu'on ne puisse tirer aucune conclusion formelle d'une expérience négative, je crois pouvoir cependant avancer l'hypothèse suivante : Les mouvements des étamines ne paraissent pas dus à des variations dans la constante capillaire des liquides contenus dans les cellules. La théorie d'IмBERT (23) sur la contraction musculaire, dérivant des travaux de Lippyanx sur les phénomènes électro-capillaires ne parait donc pas applicable aux mouvements des étamines.

Il est donc permis de conclure que l'effort direct des charges

T. LIII. (SÉANCES) 36 
électriques est nécessaire à la production du mouvement. Je rappellerai en outre, à propos de l'électro-physiologie, que d'après les expériences de Heald (21) les sucs végétaux sont des électrolytes, c'est-à-dire renferment des sels ionisés.

$3^{\circ}$ Influence de certaines substances Sur le modvement.

\section{a). Chloroforme.}

L’influence des anesthésiques est connue depuis longtemps, et les travaux de Baillon et Jourdain (1), de Kabsch (25), d'Heckel (22) et de Correns (8) sont devenus classiques. Le chloroforme, l'éther, le bromoforme, le chloral suspendent l'irritabilité. Mais cette anesthésie est temporaire, en ce sens qu'elle cesse dès que les étamines ne sont plus soumises à l'influence des anesthésiques.

Je me suis appliqué à préciser autant que possible les conditions d'action du chloroforme sur les étamines de Mahonia japonica. J'ai soumis à des vapeurs de chloroforme des fleurs pendant des temps croissants, puis lorsque les fleurs remises dans une atmosphère normale avaient repris leur sensibilité, j'ai étudié les graphiques de leurs mouvements. J'ai constaté que le fait d'avoir été anesthésiée pendant un certain temps amenait des modifications durables dans l'état de l'étamine. Les courbes obtenues étaient plus affaissées qu'avec des étamines n'ayant pas subi l'action du chloroforme et les diverses périodes du mouvement d'une durée plus grande qu'à l'état normal. Ces faits ne peuvent s'expliquer que par une altération qui persiste alors que l'anesthésie a déjà disparu. En effet un séjour un peu prolongé dans les vapeurs de chloroforme (deux heures environ amène la mort et cette mort s'accompagne d'un changement de coloration de l'étamine, et plus spécialement de l'apparition d'une tache brune sur la face interne de la base du filet, c'est-à-dire dans la région capable de réagir à une excitation mécanique.

Il semble donc que les vapeurs de chloroforme produisent une action physique, dissolvante par exemple, sur certaines des substances renfermées dans les cellules, et que l'anesthésie ne soit qu'une des manifestations de cette désorganisation des cellules motrices. 
b). Action de cerlains alcalö̈des.

L'action de la strychnine sur les plantes sensibles a fait l'objet de quelques recherches. Darwin (10) paralyse, avec l'acétate et le citrate de strychnine, les tentacules de Drosera; $\mathrm{T}_{\mathrm{Ass}}(48)$ en soumettant des fleurs à l'action de la strychnine, vit les pétales s'écarter et devenir horizontaux. Mais les recherches les plus importantes dans ce sens sont dues au physiologiste italien Borzi $(2,3)$. Cet auteur a expérimenté l'action de la strychnine et de la brucine sur les stigmates de Martynia, et les feuilles de Mimosa pudica. Il a vu que la strychnine avait un effet convulsivant, déterminant un état tétanique qui peut durer neuf jours. Le chloroforme, la cocaïne, la paraldéhyde font cesser cet état tétanique et réapparaître l'irritabilité.

J'ai fait des recherches analogues sur les étamines de diverses Berbéridées, en employant le sulfate de strychnine et le chlorhydrate de cocaïne aux doses de 1 p. 100 ou 1 p. 1000 . Mes résultats diffèrent complètement de ceux que Bonzı a obtenus sur d'autres plantes il est vrai.

La technique que j'ai employée est la suivante : on peut faire tomber dans la fleur une goutte de la dissolution de l'alcaloïde employé, mais la pénétration dans les tissus de l'étamine est lente et incertaine. Il vaut mieux isoler les six étamines d'une fleur et les placer dans la dissolution de telle sorte que la partie sectionnée du filet plonge dans le liquide.

Voici les résultats obtenus par la seconde méthode par exemple. Des étamines ont été placées simultanément dans l'eau distillée, la dissolution de sulfate de strychnine à 1 p. 1000 ou à 1 p. 100 et dans le chlorhydrate de cocaïne, toutes les autres eonditions restent égales. Examinées quatre heures après, les trois séries d'étamines sont irritables et ne paraissent pas avoir subi de modifications (dans les expériences de Borzi (3) la tétanisation des stigmates de Martynia, a eu lieu dix minutes après l'apport de strychnine). Six heures après, les étamines placées dans l'eau et la cocaïne n'ont subi aucune altération et sont encore irritables. Au contraire les étamines placées dans la strychnine étaient contractées, colorées en brun et mortes. En effet, contrairement à l'expérience de Borzı, il m’a été impossible 
de faire cesser cet état de contraction, mème dès son apparition, et de rendre les étamines irritables à nouveau, soit par le chloroforme, soit par la cocaïne. Douze heures plus tard, c'est-à-dire en tout vingt-deux heures après le début de l'expérience, les étamines placées dans la cocaïne présentent exactement le même état que celles qui ont subi l'action de la strychnine; elles sont rabattues, mortes et colorées en brun.

J'ai refait un très grand nombre de fois ces expériences, jamais je n'ai rien trouvé de comparable à ce qui a été décrit par Borzi.

La seule conclusion possible est donc celle-ci : la strychnine tue les étamines beaucoup plus vite que la cocaïne, mais ces deux substances n'ont aucune influence sur l'irritabilité el la production du mouvement. Les conséquences que Borzi a tirées de ses expériences sur les Martynia et les Sensitives ne s'appliquent done pas aux mouvements des étamines de Mahonia et de Berberis.

En ce qui concerne l'influence des autres substances, gaz, solutions salines, etc., sur les mouvements, je renvoie le lecteur aux travaux d'Heckel (22) et surtout de Corress (8) qui a rectifié et étendu les travaux de ses prédécesseurs, de $\mathbf{K}_{\text {AвSCH }}(\mathbf{2 5})$ en particulier.

\section{Considérations générales.}

Un nombre considérable de travaux, ou mieux d'hypothèses, ont été publiés sur la connaissance intime du mécanisme des mouvements provoqués. Les recherches les plus connues sont celles de Preffer $(39,42)$ qui dans de nombreux Mémoires sur les Sensitives, les étamines des Cynarées, admet qu'un contact détermine une contraction de certaines cellules, qui chassent l'eau qu'elles renferment dans les méats intercellulaires. Cette eau rompt l'équilibre physique des tissus, le mouvement se transmet ainsi de proche en proche et finalement se traduit par l'émission d'une gouttelette d'eau s'échappant par la section du filet staminal. Cette théorie n'est pas soutenable, car on ne voit jamais sortir d'eau d'une étamine sectionnée et irritée. Cela, Heckel (22) puis Chauveado (6) l'ont dit, et je l'ai maintes fois vérifié. Je n'insiste pas, dans cet aperçu biblographique, sur les travaux des élèves de Preffer, Micheli (34), Oliver (38), 
Cunningham (9), etc., qui reproduisent purement et simplement la théorie du physiologiste allemand. En somme, ce qui reste vrai de la théorie de Prefrer c'est simplement ceci, que le protoplasma des cellules du filet staminal est irritable et contractile. C'est sur ce phénomène que je vais insister en laissant de côté la très vague théorie émise par Sxetzlér (46) et appliquée récemment par Ewart (14) à d'autres cas, qui attribue le mouvement à des différences de tension entre les deux faces d un organe.

Longtemps avant Pfeffer, Сонх (7) admit que la contraction du protoplasma était la cause essentielle du mouvement. Heckel (22), dans sa thèse, développa largement cette idée et indiqua que dans les étamines contractées, le protoplasma des cellules de la face sensible était condensé autour du noyau. Il attribua le mouvement au raccourcissement ainsi obtenu sur la face ventrale, par suite de la plus grande contraction des cellules dans leur sens longitudinal. Voici les phrases mèmes d'HескеL $(22$, v. page 42$)$ :

"Comme on peut le voir, les cellules ont éprouvé un mouvement de contraction, et non pas seulement, comme le veut Pfeffer, une soustraction d'eau par les pores, car les membranes d'enveloppe présentent des plis transversaux bien accusés pendant que les cellules ont perdu au total 1/6 de longueur, ainsi qu'il résulte de nombreuses mensurations faites au micromètre tant dans des Berberis que dans les Mahonia, où les phénomènes sont identiques. Le protoplasma a quitté les parois de la cellule et semble s'ètre condensé au centre, en ne laissant entre le noyau et la membrane d'enveloppe que quelques traînées de nature granuleuse peu apparente. - Tout le contenu cellulaire (amidon, granules colorées, etc.) a suivi le protoplasma dans son mouvement rétractif... Sitôt que l'irritation de quelque nature qu'elle soit s'étant produite, toutes les cellules du parenchyme de la face concave comprises entre la courbe extérieure et la ligne diamétrale se sont contractées et que la projection de l'organe s'est produite avec raccourcissement et épaississement de toute sa masse, ces cellules tendent à revenir à leur position de repos, et leur contenu quitte le centre pour s'appliquer sur les parois. Elles sont aidées à ce retour par l'action des cellules antagonistes de la face dorsale, qui, distendues par le fléchissement de la face concave, tendent, en vertu de leur élasticité, à revenir à leur position première ".

Cette notion d'irritabilité et de contractibilité du protoplasma a été développée après les travaux d'HескеL de plus en plus. La découverte par Oliver (38), Klebs (28), Gardiner (16), Russow (45), des communications protoplasmiques de cellule à cellule, 
étudiées récemment avec grand détail par Strasburger (47), Kıenitz-Gerloff $(26,27), K_{0 H L}(29)$, donne à la notion d'irritabilité et de propagation des excitations, une base anatomique importante. Haberlandt $(19,29)$ considère comme de véritables organes du tact, les grosses cellules épidermiques qui tapissent la face ventrale du filet staminal des Berbéridées dans la région sensible. Je rappelle en outre que $\operatorname{Borzi}(4,5)$ et Fitring $(15)$ en étudiant les feuilles de Sensitive, les vrilles de Cucurbitacées, ont édifié la notion d'un système périphérique sensible et d'un système moteur central, capables de déterminer et de produire le mouvement; par exemple, ils décrivent des cellules motrices fusiformes à noyau très allongé, comparables à celles que l'on observe dans le tissu moteur des étamines mobiles.

Ces diverses théories, basées uniquement sur des structures anatomiques, ne tendent rien moins qu'à supposer chez les végétaux, l'existence d'un système nerveux, rudimentaire, sensible et moteur. La découverte des neuro-fibrilles par NEMEC $(35,36,37)$ aurait dû apporter à ces théories, un appui important. Malheureusement ces neuro-fibrilles, découvertes par NEMEc dans les organes géotropiques, n'ont jamais été observées par Haberlandt $(17,18)$ là où l'on devrait au contraire les rencontrer le plus abondamment, c'est-à-dire dans les étamines mobiles.

Si j'ajoute, en outre, qu'entre NeMEc et HaberLandt la discussion est toujours ouverte sur la nature même de ces neuro-fibrilles, on verra facilement que toutes les explications basées sur l'anatomie, seules, sont insuffisantes à résoudre les problèmes physiologiques.

Dans le même ordre d'idées Chauveaud (6) a cherché l'explication du mouvement des étamines de Berberis. Il a étudié comparativement des étamines fixées à l'état de mouvement et à l'état de repos par des vapeurs d'acide osmique. Il a vu dans l'épiderme de la face sensible un protoplasma qui noircissait par l'acide osmique et qui se déformait quand on passait de l'état de repos à l'état de mouvement. Il a conclu que ce protoplasma était à la fois sensible et moteur. En elle-mème l'observation est vraie et mes préparations ont reproduit les aspects décrits par Chauveaud. Mais son interprétation me parait 
inexacte, en ce sens qu'il est impossible d'admettre que les contractions de ce protoplasma épidermique seul produisent le mouvement. Pour établir ma manière de voir, il suffit d'enlever cet épiderme avec la lame d'un scalpel, ou de le détruire par une cautérisation superficielle. Si l'hypothèse de Chauveaud était entièrement vraie, l'étamine ainsi mutilée serait incapable d'accomplir le moindre mouvement. Or il n'en est rien, j’ai pu en effet sur des étamines ainsi débarrassées de l'épiderme de leur face sensible, produire des mouvements, plus restreints comme amplitude que dans le cas d'étamines intactes, mais qui néanmoins apparaissent avec une netteté parfaite. Je ne nie pas que l'épiderme de la face sensible ne joue un rôle dans la production du mouvement, mais je ne crois pas qu'à lui seul cet épiderme détermine le mouvement. Heckes, bien avant moi, était déjà arrivé à une conclusion analogue. Chauveadd parle en outre d'une différenciation du protoplasma de cet épiderme, qui se colore en noir par l'acide osmique. J'ai pu m'assurer par l'action de l'eau oxygénée, et les réactifs des corps gras, que cette différenciation était due simplement à l'accumulation de matières grasses en grande abondance. Cela d'ailleurs n'est pas spécial aux Berbéridées, beaucoup d'étamines non mobiles d'autres plantes possédant aussi des réserves d'huiles plus ou moins colorées dans leurs tissus.

Pour ma part, j'ai pratiqué dans des étamines mobiles de Berbéridées un grand nombre de coupes, j'ai employé tous les fixateurs possibles, un grand nombre de colorations, j'ai même essayé les méthodes de Golgi, d'Apathy, etc., le résultat a été toujours des plus minimes et dans le cas où des déformations compatibles avec le mouvement ont été observées, il m'a été impossible de savoir où était la cause et où était l'effet.

Les seuls faits que j'ai observés sont les suivants : L'épiderme de la face sensible et le tissu à petites cellules sous-jacent (tissu moteur?) renferment beaucoup de corps gras. Les cellules, sauf celles de l'épiderme, en sont petites, allongées suivant l'axe de l'étamine, à parois minces, avec quelques méats, et munis de noyaux très allongés, presque fusiformes. Le protoplasma des cellules de ce tissu fixe énergiquement les couleurs basiques d'aniline et il est très difficile de réussir les colorations 
nucléaires régressives (Safranine-Wasserblau, Bleu polychrometanin orange, violet de Rosaniline-alcool chlorhydrique, etc.).

Dans les étamines fixées à l'état de mouvement et comparées, soit à des étamines chloroformées, ou fixées à l'état de repos par le procédé de Chauveaud (6), le protoplasma est condensé autour du noyau, dans le tissu qui occupe la face ventrale du filet. Heckel, bien avant moi, avait observé ce fait, et Darwin (11) l'a signalé dans le renflement moteur des Mimosa.

C'est là le seul fait anatomique que je retiendrai et si je laisse de côté les communications protoplasmiques ou plasmodesmes qui ont été bien mises en évidence dans les étamines mobiles par Gardiner (16) nous voyons qu'il y a lieu de revenir purement et simplement à la théorie d'Heckel que j'ai énoncée précédemment. Je la résume en quelques mots.

Les tissus de la face concave et sensible des étamines mobiles de Berbéridées, sont formés de cellules à protoplasma irritable et contractile; sous l'influence d'une excitation mécanique, physique ou chimique, le protoplasma de ces cellules se contracte et se condense autour du noyau.

De cela, résulte une diminution dans la longueur de ces cellules, qui entrainant un raccourcissement de la face concave, amène le mouvement d'aller. Le mouvement de retour s'accomplit par une détente résultant de ce que le protoplasma reprend sa forme normale et les cellules leurs dimensions normales de repos.

La théorie du mouvement des étamines de Berbéridées se ramène donc à la notion d'un protoplasma irritable et capable de se contracter autour du noyau.

Mais les expressions, irritable, contractile, sont simplement des mots destinés à cacher notre ignorance. J'ai donc cherché s'il n’était pas possible de ramener la contractilité du protoplasma végétal à des actions physico-chimiques. J'ai pu dans ce sens échafauder une hypothèse que je vais exposer. Pour cela je m'appuierai sur les résultats que l'électro-physiologie et l'action du chloroforme m'ont amené à exposer dans les paragraphes précédents, ainsi que sur les idées nouvelles que LoEB (30) et ses élèves, Matrhews (32) en particulier, ont introduit dans la physiologie des nerfs et des muscles. On sait que la conclusion essentielle à laquelle sont arrivés ces auteurs est que 
les mouvements musculaires déterminés par des nerfs plongés dans des dissolutions salines sont dus aux charges électriques portées par les ions de la dissolution et surtout aux charges négatives apportées par les anions.

Or le protoplasma peut ètre considéré comme formé de substances colloïdes en petites masses, baignant dans un liquide. On peut admettre avec Matrhews que ces masses collö̈des portent des charges positives, cela gràce aux cathions qui les constituent. Ces charges maintiennent séparées ces masses colloïdes, c'est-à-dire les tiennent en dissolution. Le liquide est chargé de masses négatives, neutralisées par les précédentes. Supposons qu'un courant électrique vienne à s'établir dans ces cellules. Une charge électrique, négative par exemple, agissant en un point de l'étamine amène l'agglutination des masses colloïdes, et comme leurs surfaces diminuent par rapport à leur volume, leurs charges positives diminuent.

Par suite les charges négatives sont libérées dans le dissolvant. Ces tranches négatives agissant à leur tour sur les tranches colloïdes suivantes, les agglutinent et de proche en proche le phénomène se propage dans une même cellule, puis de cellule à cellule par les plasmodesmes, entraìnant l'agglutination, c'està-dire la condensation du protoplasma et le mouvement. Je rappelle que l'anatomie nous a révélé l'existence de cette agglutination. Les excitants mécaniques, chimiques, etc., agissent de mème en produisant au point irrité l'agglutination des colloides. De telle sorte, qu'en dernière analyse, on peut dire que toutes les excitations sont d'ordre électrique, et que cela est dù à l'état d'ionisation des éléments constitutifs du contenu cellulaire.

Dès lors l'action du chloroforme s'explique très simplement. Les vapeurs de chloroforme liquéfient les substances colloïdes du protoplasma et rendent l'agglutination impossible. Si l'action du chloroforme est de courte durée, la liquéfaction est incomplète et l'agglutination peut se produire de nouveau. Si elle est prolongée, la liquéfaction est complète et la cellule désorganisée est morte. C'est ce que l'expérience directe nous avait appris.

En résumé, dans ce travail j'ai fait : $1^{\circ}$ l'étude analỵtique 
du mouvement des étamines de Berbéridées; $2^{\circ}$ j’ai établi les lois de l'électro-physiologie et montré que vraisemblablement les phénomènes électro-capillaires n'interviennent pas d'une façon simple et que l'effort direct de charges électriques était nécessaire à la production du mouvement; $3^{\circ}$ j'ai précisé l'action des anesthésiques et montré que les travaux de Borzi sur l'action de la strychnine ne sont pas susceptibles de généralisations.

J'ai enfin montré que la théorie d'Heckes, qui considère la contraction du protoplasma dans les cellules du tissu de la face concave sensible comme cause directe du mouvement, était seule compatible avec les faits. J'ai établi, en dernier lieu, que seule une hypothèse basée sur des actions électriques était capable dans l'état actuel de la science de rendre compte des phénomènes de contraction protoplasmique et des mouvements. qu'ils déterminent.

Postérieurement à la rédaction de ce travail, j'ai eu connaissance de deux publications importantes qui confirment les idées que j'ai exposées. Ces deux Mémoires qui ne figurent pas dans la liste bibliographique générale sont les suivants :

Linsbauer (K.) - Zur Kenntniss der Reizbarkeit der Centaureafilamente. Sitz. d. Kais. Akad. de Wiss. Wien math. nat. Cl. 114, 1905.

BösE (J.-C). - Plant response as a means of physiological investigation. Londres, 1906.

Lissbauer a étudié les phénomènes de mouvement présentés par les étamines de diverses Centaurea américains. Il a décrit dans le mouvement une période d'excitation latente de 1 seconde, une contraction d'une durée de 7 à 13 secondes et une troisième période de 50 à 60 secondes pendant laquelle l'étamine revient dans sa position normale et au bout de laquelle elle est de nouveau contractile. On remarquera en particulier que le rapport de la durée d'excitation latente à la durée de contraction est d'environ 1 dixième, c'est-à-dire le mème que celui que j'ai signalé dans le mouvement beaucoup plus rapide des étamines des Berbéridées. Linsbader montre en outre que les poils qui garnissent l'étamine des Centaurées, ne sont pas des organes de perception comme l'avançait Haberiandt $(19,20)$. Tout au plus ce seraient des stimulateurs, la sensibilité et la motilité résidant dans le parenchyme staminal lui-mème. Peutêtre l'épiderme de la face sensible des étamines des Berberis, et surtout les grosses celluleuses papilleuses de la base, jouent-ils un rôle analogue aux poils stimulateurs des Centaurées. 
Quant au travail de Böse, il porte sur la généralité du phénomène de contraction protoplasmique sous l'influence d'une irritation physique ou chimique. Böse, montre en effet que c'est là la réaction normale des tissus végétaux aux excitations extérieures.

\section{Bibliographie}

1. Baillon et Jourdain. - C. R. Ac. Sciences, t. LXX. 1870.

2. BoRzI. - Contribu. alla conose. de fenomeni di sensibilita delle piante. - Palerme, 1883.

3. - Action de la strychnine et de la brucine sur les organes sensibles des plantes. - Archives ital. de biologie. 32. 1899.

4. - L'apparato di moto delle Sensitive. - Rev. sc. Biol. 4. 1899.

5. - Anatomie del apparato senso-motore dei cirri delle Cucurbitacees. Contrib. alla Biol. veget. vol. III. Palerme, 1902.

6. Chauveaud. - C. R. Acad. Sciences CXIX. 1901. Bullet. Mus. Hist. Nat. 1901.

7. CoHs. - Contractile gewebe in Pflanzenreich. - Jahrbuch der Schlen. Gesell. fur vaterland Cultur. 1861.

8. ConRens. - Uber die Abhändigkeit der Reizerscheinungen höherer Pflanzen von der Gegenwart freien Sauerstoffes. - Flora 1892.

9. Cunningham. - On the phenomena of propagation mov $v^{t}$ in Mimosa pudica. Scientific mem. by medical off. of the army of India. Part. III. Calcutta, 1887.

10. DARWIN. - Les plantes insectivores.

11. - La faculté motrice dans les plantes.

12. Dewevre et Bordage. - Sur l'analyse photographique des mouvements des végétaux. - Rev. gén. de Botanique. IV. 1892.

13. Dop (P.) - Physiologie des mouvements des eitamines de Mahonia nepalensis. Bull. Soc. Bot. de France, $4^{\mathrm{e}}$ série, t. V. 1905.

14. Ewart. - On contact irritability. - Annal. du Jard. Bot. de Buitenzorg V. XV (1898).

15. FitTing. - Weitere Untersuchungen zur Physiologie der Ranken, nebst einigen neuen versuchen über die Reizleitung bei Mimosa. - Iahrb. fur wiss. Bot. Bd. XXXIX. -1903.

16. GaRDINER. - On open communication between the cells in the pulvinus of Mimosa Q. J. Micros. Soc. 1882. - Philosophical transact. Roy. Society. III. 1883.

17. Haberlandt. - Uber fibrillaren Plasmastructuren. - Berichte d. D. Bot. Ges. XIX. 1901.

18. - Uber Rcizleitung in Pflanzenreich. - Biol. Centralblat XIX. 1904.

19. - Sinnes organe in Pflanzenreich zur Perception mechanischer Reize. Liepzig, 1901.

20. - Physiologische Pflanzenanatomie. 1904.

21. HeaLD. - The electrical conductibility of plantes juices, Bot. gaz. XXXIV.

22. Heckel. - Du mouvement végétal. Thèse Doct. ès sciences. Montpellier, 1875 .

23. Imbert. - Archives de physiologie. 1897.

24. Iurgensen. - Studien des Phys. Institut zu Breslau, 1861. 
25. KaBsch. - Anatomische und physiolog. Beobachtungen uber die Reizbarkeit der Geschlets organe. Bot. Zeitung. 1861.

26. KiEnitz-GerlofF. - Die Protoplasma verbindungen zwischen benachbarten Gewebselementen in der Pflanzen, Bot. Zeitung. 1891.

27. Kienitz-Gerloff. - Neue Stüdien uber Plasmodesmen. - Berichte der D. Bot. Ges Bd. XX. 1902.

28. KLEBS. - Botanische Zeitung. 1884.

29. Kонl. - Beiträge zur Kentniss der Plasmaverbindunyen in der Pflanzen. Beihefte z. Bot. Centralblatt Bd. XII. 1902.

30. Lовв. - The physiological effects on the electrical charge of ions, and the electrical character of life. Meet. Amer. Physiol. Soc. 1903. Chicago.

31. MAC-FARLane. - Irrito-contractility in plants. Biolog. lectures delivred at the marine biol. laboratory of Woods Hell. Lit. III. Boston, 1894.

32. MatThews. - The nature of nerve stimulation and of charges in irritability. (Sc. N. S. 1902).

33. - The nature of nerve irritability and of chemical and electrical stimulus. - Sc. N. S. XVII.

34. Micheli. - Arch. des Sc. phys. et natur. Genève, 1895.

35. Nemec. - Die Reizleitende Struckturen bei den Pflanzen. Biol. Centralb. Bd. XX. 1900.

36. Die Reizleitung und reizleitenden Struckturen bei den Pflanzen. Iéna 1901. 37. - Die Bedeutung der fibrillaren Struckturen bei den Pflanzen. Biol. Centr. XXI. 1901.

38. OLIVER. - Uber Fortleitung des Reizes bei reizbaren Narben. - Ber. der D. Bot. Ges. V. 1887.

39. Pfeffer. - Physiologische Untersuchungen, 1873 .

40. - Uber Fortpflanzung der Reize. - Iahrb. f. voiss. Bot. Bd. IX.

41. - Die Reizbarkeit der Pflanzen. 1893.

42. - Zur Kenntniss der Plasmahaut und der vacuolen nebst Bemerkungeu über die Aggregat zustand des protoplasmas und über osmotiche Vorgänge. - Abh. der math. - phys. classe der Kon. Sachs. Ges. der Wissemchaften. 1890.

43. - Pflanzenphysiologie; traduct. Friedel. 1904-1906.

44. Rina-ScotT. - On the movements of the Flowers of Sparmannia africana. Ann. of Botany. 1903.

45. Russow - Sitz. der Dorpater. Nat Gesell. 1882.

46. Snetzler. - Bulletin de la Soc. Vaudoise des Sc. nat. L. X. 1869.

47. Strasburger. - Uber Plasma verbindungen pflanzlicher Zeller. Iahr.

f. wiss. Bot. 1901 .

48. Tassi. - Gli effecti anesteci sui fiori. Sienne, 1884.

M. Gagnepain donne, au nom de M. Finet et au sien, lecture de la communication suivante : 


\section{$2 \mathrm{BHL}$ Biodiversity Heritage Library}

Dop, Paul. 1906. "Recherches physiologiques sur le mouvement des étamines des Berbéridées." Bulletin de la Société botanique de France 53, 554-572. https://doi.org/10.1080/00378941.1906.10831203.

View This Item Online: https://www.biodiversitylibrary.org/item/8675

DOI: https://doi.org/10.1080/00378941.1906.10831203

Permalink: https://www.biodiversitylibrary.org/partpdf/160597.

\section{Holding Institution}

Missouri Botanical Garden, Peter H. Raven Library

\section{Sponsored by}

Missouri Botanical Garden

\section{Copyright \& Reuse}

Copyright Status: Public domain. The BHL considers that this work is no longer under copyright protection.

This document was created from content at the Biodiversity Heritage Library, the world's largest open access digital library for biodiversity literature and archives. Visit BHL at https://www.biodiversitylibrary.org. 